\title{
Scaling of PDFs, TMDs, and GPDs in soft-wall AdS/QCD
}

\author{
Valery E. Lyubovitskij $\oplus^{1,2}$ and Ivan Schmidt $\odot^{2}$ \\ ${ }^{1}$ Institut für Theoretische Physik, Universität Tübingen, Kepler Center for Astro and Particle Physics, \\ Auf der Morgenstelle 14, D-72076 Tübingen, Germany \\ ${ }^{2}$ Departamento de Física y Centro Científico Tecnológico de Valparaíso-CCTVal, \\ Universidad Técnica Federico Santa María, Casilla 110-V, Valparaíso, Chile
}

(Received 22 May 2020; accepted 24 July 2020; published 11 August 2020)

\begin{abstract}
We explicitly demonstrate how to correctly define the hadronic parton distributions [parton distribution functions (PDFs), transverse-momentum-dependent parton distribution functions, and generalized parton distributions] in the soft-wall anti-de Sitter/QCD approach, based on the use of a quadratic dilaton field, providing confinement and breaking of conformal and chiral symmetries. The power behavior of parton distributions at large values of the light-cone variable is consistent with quark counting rules and Drell-YanWest duality. All parton distributions are defined in terms of profile functions, which depend on the lightcone coordinate and are fixed from PDFs and electromagnetic form factors.
\end{abstract}

DOI: $10.1103 /$ PhysRevD.102.034011

\section{INTRODUCTION}

During the last decade the soft-wall anti-de Sitter/QCD [1-3] formalism achieved significant progress in the description of hadron structure: mass spectrum, parton distributions, form factors, etc. (for an overview, see, e.g., Ref. [4]), based on an effective action constructed with the use of a quadratic dilaton field providing confinement and breaking of both conformal and chiral symmetry. This dilaton field $\varphi(z)$ has quadratic dependence on the holographic variable $z$, and is multiplied with the dilaton scale parameter $\kappa$ (of order of a few hundreds of $\mathrm{MeV}$ ): $\varphi(z)=\exp \left(-\kappa^{2} z^{2}\right)$. Chiral symmetry breaking can be achieved in the soft-wall AdS/QCD model by using a modified dilaton profile and a quartic term in the bulk scalar potential. Such a modification allows one to separate the dependence on spontaneous and explicit chiral symmetry breaking. One should stress that in Ref. [5] the issue of chiral symmetry breaking was further studied and understood. In particular, in [6] a new class of holographic models has been derived in the so-called Veneziano limit, in which the numbers of both flavors and colors are large $N_{f}, N_{c} \rightarrow \infty$ and their ratio $N_{f} / N_{c}$ is fixed. In this approach, chiral symmetry breaking is ruled out by the running of the anomalous dimension of the chiral condensate and the Breitenlohner-Freedman bound violation.

Published by the American Physical Society under the terms of the Creative Commons Attribution 4.0 International license. Further distribution of this work must maintain attribution to the author(s) and the published article's title, journal citation, and DOI. Funded by SCOAP ${ }^{3}$.
Soft-wall AdS/QCD is a mainly phenomenological approach in the class of holographic approaches and needs further justification to be more consistent with QCD. However, there are a few advantages of this approach, which make it useful in the study of properties of hadrons and exotic states. One of its advantages is that it explicitly reproduces the power scaling of hadronic form factors at large $Q^{2}$ [4-15]. In particular, soft-wall AdS/QCD is consistent with the Drell-Yan-West (DYW) relation [16] between the large- $Q^{2}$ behavior of nucleon electromagnetic form factors and the large- $x$ behavior of the structure functions (see also Ref. [17] for the extension to inelastic scattering) and quark counting rules [18]. Based on the findings in Refs. [16-18] one can, e.g., relate the behavior of the quark distribution function [parton distribution function (PDF)] in nucleon $q_{v}(x) \sim(1-x)^{p}$ at $x \rightarrow 1$ with the scaling of the proton Dirac form factor $F_{1}^{p}\left(Q^{2}\right) \sim$ $1 /\left(Q^{2}\right)^{(p+1) / 2}$ at large $Q^{2}$, where the parameter $p$ is related to the number of constituents in the proton (or twist $\tau$ ) as $p=2 \tau-3[16,19]$. At large $x$ and finite $Q^{2}$ there are also model-independent predictions of perturbative QCD (pQCD) for the generalized parton distributions (GPDs) [20]—pion $\mathcal{H}_{q}^{\pi}\left(x, Q^{2}\right)$ and nucleon $\mathcal{H}_{q}^{N}\left(x, Q^{2}\right), \mathcal{E}_{q}^{N}\left(x, Q^{2}\right)$ :

$\mathcal{H}_{q}^{\pi}\left(x, Q^{2}\right) \sim(1-x)^{2}, \quad \mathcal{H}_{q}^{N}\left(x, Q^{2}\right) \sim(1-x)^{3}$, $\mathcal{E}_{q}^{N}\left(x, Q^{2}\right) \sim(1-x)^{5}$.

Note that the prediction of $\mathrm{pQCD}$ for the pion PDF $q_{\pi}(x) \sim(1-x)^{2}$ at large $x$ (it trivially follows from the prediction for GPDs [20]) was supported by the updated analysis [21] of the E615 data [22] on the cross section of the Drell-Yan (DY) process $\pi^{-} N \rightarrow \mu^{+} \mu^{-} X$, including 
next-to-leading logarithmic threshold resummation effects: $q_{\pi}(x) \sim(1-x)^{2.03}$ at the initial scale $\mu_{0}=0.63 \mathrm{GeV}$ [21].

Parton distributions in hadrons play an important role in the QCD description of hadrons and in their interactions in terms of quarks and gluons. In particular, QCD factorization allows one to separate effects of strong interactions at small distances (perturbative dynamics of quark and gluons) from long-distance (or small momenta) effectsnonperturbative part. This last part is parametrized by parton distribution functions, which are universal functions for each hadron and independent of the specific process. In this vein one can represent observable quantities, such as cross sections, as having both perturbative and nonperturbative pieces. The perturbative part of the cross section is defined by those subprocesses which come from the hard interactions of quarks, gluons, and electroweak particles and which can then be calculated perturbatively using the Standard Model. The nonperturbative part is encoded in parton distributions, which cannot be directly calculated in QCD, and therefore different theoretical approaches (world data analysis, lattice and light-front QCD, quark and potential models, etc.) have been applied to extract or predict the PDFs, TMDs, and GPDs (for a recent overview see, e.g., Ref. [23]).

The importance of the scaling laws and their role in the description of nucleon structure has been stressed and studied in detail in the literature. Moreover, they are important for the proper construction of light-front (LF) QCD approaches [4,7,24-31], motivated by soft-wall AdS/QCD and developed in the past decade. The main advantage of these LF QCD approaches was in the construction of effective wave functions for mesons $[4,7,10,11,24,27,30-32]$, baryons [25,26,28-31], and hadrons with an arbitrary number of partons (arbitrary twist) $[25,26,28,30,31]$, which were further used in the calculation of fundamental properties of hadrons-parton distributions and form factors. While form factors and parton distributions in LF QCD were consistent with quark counting rules at large $Q^{2}$ and large $x \rightarrow 1$ (light-cone variable), there was the problem of achieving full consistency in soft-wall AdS/QCD. As we stressed before, hadronic form factors in soft-wall AdS/QCD obey the power scaling $1 / Q^{2(\tau-1)}$ at large $Q^{2}$ and for arbitrary twist $\tau$ of a hadron. On the other hand, parton distributions (like PDF and GPDs) calculated in soft-wall AdS/QCD (see, e.g., Refs. $[7,9,12])$ have different scalings at large $x$. In particular, the pion PDF scaled as $(1-x)^{0}[7,12]$, the nucleon charged and magnetization PDFs/GPDs are scaled as $(1-x)$ and $(1-x)^{2}$, respectively [9]. Such behavior of PDFs was obtained starting from the effective actions for mesons and baryons with total angular spin $J$ [4-15] (see in the next section discussion of these actions and derivation of the PDFs/GPDs from them).

In Refs. [7,9,12] the integral representation for the hadronic form factor with twist $\tau$ has been derived, which can also be written in closed form as the beta function $B(\alpha, \beta)$,

$$
\begin{aligned}
F_{\tau}\left(Q^{2}\right) & =\int_{0}^{1} d y(\tau-1)(1-y)^{\tau-2} y^{a} \\
& =(\tau-1) B(\tau-1, a+1) .
\end{aligned}
$$

Using identification of the $y$ variable with the lightcone momentum fraction $x$ both PDFs $q_{\tau}(x)$ and GPDs $\mathcal{H}_{\tau}\left(x, Q^{2}\right)$ have been extracted $[7,9,26]$ :

$$
q_{\tau}(x)=(\tau-1)(1-x)^{\tau-2}, \quad \mathcal{H}_{\tau}\left(x, Q^{2}\right)=q_{\tau}(x) x^{a} .
$$

Such $x$ dependence of PDF and GPD contradicts modelindependent results: the DY inclusive counting rule for $q_{\tau}(x)$ at $x \rightarrow 1[16,19,20]$ and the prediction of $\mathrm{pQCD}$ for GPDspion $\mathcal{H}_{q}^{\pi}\left(x, Q^{2}\right)$ and nucleon $\mathcal{H}_{q}^{N}\left(x, Q^{2}\right), \mathcal{E}_{q}^{N}\left(x, Q^{2}\right)$ at large $x$ and finite $Q^{2}$ [20].

It was first noticed in Ref. [25] that the interpretation of the variable $y$ in the integral representation (25) as lightcone variable is not truly correct and that one can think about a generalized light-cone variable $y(x)$ depending on $x$. Then the power behavior of hadronic PDFs and GPDs ar large $x$ is consistent with model-independent results of Refs. $[16,19,20]$ can be obtained, provided that an appropriate choice of the $x$ dependence of the function $y(x)$ is made. In particular, the simplest choice the function $y(x)$ was found as

$$
y_{N}(x)=\exp \left[-\log (1 / x)(1-x)^{2 /(N-1)}\right]
$$

leading to the correct large- $x$ scaling of PDFs and GPDs in mesons

$$
q_{\tau}^{M}(x) \sim \mathcal{H}_{\tau}^{M}\left(x, Q^{2}\right) \sim(1-x)^{2 \tau-2}
$$

at $N=2 \tau-2$ and in baryons

$$
q_{\tau}^{B}(x) \sim \mathcal{H}_{\tau}^{B}\left(x, Q^{2}\right) \sim(1-x)^{2 \tau-3}
$$

at $N=2 \tau-3$. The function $y_{\tau}(x)$ obeys the following boundary conditions $y_{\tau}(0)=0$ and $y_{\tau}(1)=1$. Notice that a similar idea was recently considered in the framework of light-front holographic QCD (LFHQCD) [30,31] (see also Ref. [33] for an extension of Ref. [30]). In particular, a function [named as $w(x)$ ] was introduced in the integral representation of the form factor [30,31]:

$$
F_{\tau}\left(Q^{2}\right)=\frac{1}{N_{\tau}} \int_{0}^{1} d x w^{\prime}(x)[w(x)]^{Q^{2} / 4 \lambda-1 / 2}[1-w(x)]^{\tau-2} .
$$

Obviously, the two mathematical extensions considered in Ref. [25] and Refs. [30,31] are equivalent. The only 
difference is that in Refs. [30,31] an extra power $-1 / 2$ was included in the $[w(x)]^{Q^{2} / 4 \lambda-1 / 2}$, while in the soft-wall model $[7,9,12]$ the factor is $[w(x)]^{Q^{2} / 4 \lambda}$. In other words, the soft-wall model $[7,9,12]$ and LFHQCD $[7,9,12]$ deal with slightly different analytical expressions for the hadronic form factors: $F_{\tau}\left(Q^{2}\right) \sim B\left(\tau-1,1+Q^{2} / 4 \lambda\right)$ in softwall AdS/QCD and $F_{\tau}\left(Q^{2}\right) \sim B\left(\tau-1,1 / 2+Q^{2} / 4 \lambda\right)$ in LFHQCD.

The main objective of this paper is to continue the discussion of ideas started in Refs. [25,30,31] and propose a more simple derivation of PDFs, transverse-momentumdependent parton distribution functions (TMDs), and GPDs of hadrons with arbitrary twist in the context of soft-wall AdS/QCD models. In particular, we explicitly demonstrate how to correctly define the hadronic parton distributions (PDFs, TMDs, and GPDs) in the soft-wall AdS/QCD approach, based on the use of a quadratic dilaton field providing confinement and breaking of conformal and chiral symmetry. The obtained power behavior of parton distributions at large values of lightcone variable $x$ are then consistent with quark counting rules and DYW duality. All parton distributions are defined in terms of profile functions depending on the light-cone coordinate and are fixed from PDFs and electromagnetic form factors.

The paper is organized as follows. In Sec. II we present an overview of our approach and consider derivation of PDFs. TMDs will be derived in Sec. III. In Sec. IV we discuss derivation of GPDs. Finally, Sec. V contains our summary and conclusions.

\section{PARTON DISTRIBUTION FUNCTIONS (PDFs)}

\section{A. General consideration}

We start with a brief overview of the effective action for AdS fields (bosons and fermions) dual to mesons and baryons, derived in our previous papers (for more details see, e.g., Refs. $[9,12])$. We consider the propagation of boson $\Phi_{M_{1} \cdots M_{J}}(x, z)$ and fermion $\Psi_{M_{1} \cdots M_{J-1 / 2}}(x, z)$ fields with spin $J$ (dual to mesons and baryons, respectively) in a fivedimensional AdS space. The AdS metric is specified by

$$
\begin{aligned}
d s^{2} & =g_{M N} d x^{M} d x^{N}=\eta_{a b} e^{2 A(z)} d x^{a} d x^{b} \\
& =e^{2 A(z)}\left(\eta_{\mu \nu} d x^{\mu} d x^{\nu}-d z^{2}\right), \\
\eta_{\mu \nu} & =\operatorname{diag}(1,-1, \ldots,-1),
\end{aligned}
$$

where $M$ and $N=0,1, \ldots, d$ are the space-time (base manifold) indices, $a=(\mu, z)$ and $b=(\nu, z)$ are the local Lorentz (tangent) indices, $g_{M N}$ and $\eta_{a b}$ are curved and flat metric tensors, which are related by the vielbein $\epsilon_{M}^{a}(z)=$ $e^{A(z)} \delta_{M}^{a}$ as $g_{M N}=\epsilon_{M}^{a} \epsilon_{N}^{b} \eta_{a b}$. Here $z$ is the holographic coordinate, $R$ is the AdS radius, and $g=\left|\operatorname{det} g_{M N}\right|=$ $e^{10 A(z)}$. We restrict ourselves to a conformal-invariant metric with $A(z)=\log (R / z)$, where $R$ is the $\operatorname{AdS}$ radius. The boson action is written as

$$
\begin{aligned}
S_{B}= & \frac{(-)^{J}}{2} \int d^{d} x d z \sqrt{g} e^{-\varphi(z)}\left[g^{M N} g^{M_{1} N_{1}} \cdots g^{M_{J} N_{J}} \partial_{M} \Phi_{M_{1} \cdots M_{J}}(x, z) \partial_{N} \Phi_{N_{1} \cdots N_{J}}(x, z)\right. \\
& \left.-\left(\mu_{J}^{2}+V_{J}(z)\right) g^{M_{1} N_{1}} \cdots g^{M_{J} N_{J}} \Phi_{M_{1} \cdots M_{J}}(x, z) \Phi_{N_{1} \cdots N_{J}}(x, z)\right]
\end{aligned}
$$

where bosonic spin- $J$ field $\Phi_{M_{1} \cdots M_{J}}(x, z)$ is described by a symmetric, traceless tensor, satisfying the conditions

$$
\partial^{M_{1}} \Phi_{M_{1} M_{2} \cdots M_{J}}=0, \quad g^{M_{1} M_{2}} \Phi_{M_{1} M_{2} \cdots M_{J}}=0 .
$$

Here $V_{J}(z)=e^{-2 A(z)} U_{J}(z)$, where $U_{J}(z)$ is the effective dilaton potential

$$
U_{J}(z)=\frac{1}{2} \varphi^{\prime \prime}(z)+(d-1-2 J) \varphi^{\prime}(z) A^{\prime}(z)
$$

and

$$
\mu_{J}^{2} R^{2}=(\Delta-J)(\Delta+J-4)
$$

is the bulk mass. The quadratic dilaton field $\varphi(z)$ is specified as $\varphi(z)=\kappa^{2} z^{2}$, where $\kappa$ is the dimensional parameter. The dimension of the boson AdS fields $\Delta$ is identified with twist $\tau$ as $\Delta=\tau=N+L$, where $N$ is the number of partons and $L$ is the orbital angular momentum.

Restricting to the axial gauge $\Phi_{\ldots z \ldots}(x, z)=0$ and performing the Kaluza-Klein expansion

$$
\Phi^{\mu_{1} \cdots \mu_{J}}(x, z)=\sum_{n} \Phi_{n}^{\mu_{1} \cdots \mu_{J}}(x) \Phi_{n}(z)
$$

one can derive the equation of motion (EOM) for the profile function $\phi_{n \tau}(z)=e^{3 A(z) / 2} \Phi_{n}(z)$ :

$\left[-\frac{d^{2}}{d z^{2}}+\frac{4(\tau-2)^{2}-1}{4 z^{2}}+U_{J}(z)\right] \phi_{n \tau}(z)=M_{n \tau J}^{2} \phi_{n \tau}(z)$

with analytical solutions for the bulk profile

$$
\phi_{n \tau}(z)=\sqrt{\frac{2}{\Gamma(\tau-1)}} \kappa^{\tau-1} z^{\tau-3 / 2} e^{-\kappa^{2} z^{2} / 2} L_{n}^{\tau-2}\left(\kappa^{2} z^{2}\right)
$$


and mass spectrum

$$
M_{n \tau J}^{2}=4 \kappa^{2}\left(n+\frac{\tau+J}{2}-1\right) .
$$

Here $L_{n}^{m}(x)$ are the generalized Laguerre polynomials.

In the case of AdS fermion fields $\Psi_{K_{1} \cdots K_{J-1 / 2}}(x, z)$ with spin $J$, the action reads [12]

$$
\begin{aligned}
S_{F}= & \int d^{d} x d z \sqrt{g} e^{-\varphi(z)} g^{K_{1} N_{1}} \cdots g^{K_{J-1 / 2} N_{J-1 / 2}}\left[\frac{i}{2} \bar{\Psi}_{K_{1} \cdots K_{J-1 / 2}}(x, z) \epsilon_{a}^{M} \Gamma^{a} \mathcal{D}_{M} \Psi_{N_{1} \cdots N_{J-1 / 2}}(x, z)\right. \\
& \left.-\frac{i}{2}\left(\mathcal{D}_{M} \Psi_{K_{1} \cdots K_{J-1 / 2}}(x, z)\right)^{\dagger} \Gamma^{0} \epsilon_{a}^{M} \Gamma^{a} \Psi_{N_{1} \cdots N_{J-1 / 2}}(x, z)-\bar{\Psi}_{K_{1} \cdots K_{J-1 / 2}}(x, z)\left(\mu+V_{F}(z)\right) \Psi_{N_{1} \cdots N_{J-1 / 2}}(x, z)\right],
\end{aligned}
$$

where $V_{F}(z)=\varphi(z) / R$ is the dilaton potential, $\mathcal{D}_{M}$ is the covariant derivative acting on the spin-tensor field $\Psi_{N_{1} \cdots N_{J-1 / 2}}^{ \pm}$as

$\mathcal{D}_{M} \Psi_{N_{1} \cdots N_{J-1} / 2}=\partial_{M} \Psi_{N_{1} \cdots N_{J-1 / 2}}-\frac{1}{8} \omega_{M}^{a b}\left[\Gamma_{a}, \Gamma_{b}\right] \Psi_{N_{1} \cdots N_{J-1 / 2}}$,

where $\omega_{M}^{a b}=A^{\prime}(z)\left(\delta_{z}^{a} \delta_{M}^{b}-\delta_{z}^{b} \delta_{M}^{a}\right)$ is the spin connection term, and $\Gamma^{a}=\left(\gamma^{\mu},-i \gamma^{5}\right)$ are the Dirac matrices.

After expanding the fermion field in left- and rightchirality components $\Psi^{L / R}=\left(1 \mp \gamma^{5}\right) / 2 \Psi$ and a KaluzaKlein expansion for the $\Psi^{L / R}(x, z)$ fields $\Psi^{L / R}(x, z)=$ $\sum_{n} \Psi_{n}^{L / R}(x) F_{n}^{L / R}(z)$, one can obtain decoupled Schrödinger EOMs for the fermion bulk profiles $f_{n}^{L / R}(z)=e^{2 A(z)} F_{n}^{L / R}(z)$ :

$$
\begin{aligned}
& {\left[-\partial_{z}^{2}+\kappa^{4} z^{2}+2 \kappa^{2}\left(m \mp \frac{1}{2}\right)+\frac{m(m \pm 1)}{z^{2}}\right] f_{n \tau}^{L / R}(z)} \\
& \quad=M_{n \tau}^{2} f_{n \tau}^{L / R}(z)
\end{aligned}
$$

where $m=\tau-3 / 2=L+3 / 2$ and

$$
\begin{aligned}
& f_{n \tau}^{L}(z)=\sqrt{\frac{2 \Gamma(n+1)}{\Gamma(n+\tau)}} \kappa^{\tau} z^{\tau-1 / 2} e^{-\kappa^{2} z^{2} / 2} L_{n}^{\tau-1}\left(\kappa^{2} z^{2}\right), \\
& f_{n \tau}^{R}(z)=\sqrt{\frac{2 \Gamma(n+1)}{\Gamma(n+\tau-1)}} \kappa^{\tau-1} z^{\tau-3 / 2} e^{-\kappa^{2} z^{2} / 2} L_{n}^{\tau-2}\left(\kappa^{2} z^{2}\right),
\end{aligned}
$$

and

$$
M_{n \tau}^{2}=4 \kappa^{2}(n+\tau-1)=4 \kappa^{2}(n+L+2) .
$$

In order to study electromagnetic properties of hadrons we need to calculate the vector bulk-to-boundary propagator $V(q, z)$, dual to the $q^{2}$-dependent electromagnetic current: $\partial_{z}\left(\frac{e^{-\varphi(z)}}{z} \partial_{z} V\left(-q^{2}, z\right)\right)+q^{2} \frac{e^{-\varphi(z)}}{z} V\left(-q^{2}, z\right)=0$.

The latter equation is solved analytically in terms of the gamma $\Gamma(n)$ and Tricomi $U(a, b, z)$ functions:

$$
V\left(Q^{2}, z\right)=\Gamma(1+a) U\left(a, 0, \kappa^{2} z^{2}\right),
$$

where $Q^{2}=-q^{2}$ and $a=Q^{2} /\left(4 \kappa^{2}\right)$. It is convenient to use the integral representation for $V(Q, z)$ [34]

$$
V\left(Q^{2}, z\right)=\kappa^{2} z^{2} \int_{0}^{1} \frac{d y}{(1-y)^{2}} y^{a} e^{-\kappa^{2} z^{2} \frac{y}{1-y}} .
$$

The expression for the hadron form factors in the soft-wall AdS/QCD is given by

$$
F_{n \tau}\left(Q^{2}\right)=\int_{0}^{\infty} d z \phi_{n \tau}^{2}(z) V\left(Q^{2}, z\right),
$$

where the integrand contains the square of the holographic wave function in fifth dimension $z$ (dual to hadron wave function), multiplied with the vector bulk-to-boundary propagator $V\left(Q^{2}, z\right)$.

Now we are in a position to start the derivation of PDFs in soft-wall AdS/QCD. In the following, for simplicity, we restrict to a consideration of ground states of hadrons with $n=0$. In Refs. [25,30,31], this quantity has been derived using an integral representation for the hadronic form factor (see discussion in the Introduction). The easiest way is to start with the hadronic wave function normalization condition, which depends on the holographic variable $z$ :

$$
1=\int_{0}^{1} d z \phi_{\tau}^{2}(z),
$$

where $\phi_{\tau}(z)$ is the AdS bulk profile function (for simplicity we restrict here to the bosonic case and extension to the fermion case is straightforward). Next we use the integral representation for unity 


$$
\begin{aligned}
1 & =-e^{\kappa^{2} z^{2}} \int_{0}^{1} d\left[f_{\tau}(x) e^{-\kappa^{2} z^{2} /(1-x)^{2}}\right] \\
& =e^{\kappa^{2} z^{2}} \int_{0}^{1} d x\left[\frac{2 f_{\tau}(x) \kappa^{2} z^{2}}{(1-x)^{3}}-f_{\tau}^{\prime}(x)\right] e^{-\kappa^{2} z^{2} /(1-x)^{2}}
\end{aligned}
$$

and insert it into Eq. (27). Here $x$ is the light-cone coordinate and $f_{\tau}(x)$ is the profile function with boundary condition $f_{\tau}(0)=1$, which is specific for a particular hadron and fixed from its PDF. The functions $f_{\tau}(x)$ and $y_{\tau}(x)$ [see Eq. (4)] are related as

$$
\left(1-y_{\tau}(x)\right)^{\tau-1}=f_{\tau}(x)(1-x)^{2(\tau-1)}
$$

or

$$
y_{\tau}(x)=1-\left[f_{\tau}(x)\right]^{\frac{1}{\tau-1}}(1-x)^{2} .
$$

We remind the reader that at $x=0$ the functions $y_{\tau}(x)$ and $f_{\tau}(x)$ obey the boundary conditions $y_{\tau}(0)=0$ and $f_{\tau}(0)=1$. At $x=1$ function $f_{\tau}$ is finite and its value depends on the specific choice of twist $\tau$ (see below), while $y_{\tau}(1)=1$ is independent on twist.

After integration over the variable $z$ we get

$$
1=\int_{0}^{1} d x(1-x)^{2 \tau-3}\left[2 f_{\tau}(x)(\tau-1)-f_{\tau}^{\prime}(x)(1-x)\right] .
$$

Here and in the following the superscript (') means derivative with respect to variable $x$. Using a general definition for the hadronic PDF $q_{\tau}(x)$, in the form of the integral representation (first moment) over $x$

$$
1=\int_{0}^{1} d x q_{\tau}(x)
$$

we get

$$
\begin{aligned}
q_{\tau}(x) & =(1-x)^{2 \tau-3}\left[2 f_{\tau}(x)(\tau-1)-f_{\tau}^{\prime}(x)(1-x)\right] \\
& =\left[-f_{\tau}(x)(1-x)^{2 \tau-2}\right]^{\prime} .
\end{aligned}
$$

We require that the hadronic $\operatorname{PDF} q_{\tau}(x)$ must have the correct scaling at large $x$ and this behavior is governed by the profile function $f_{\tau}(x)$.

\section{B. Pion PDF}

Now let us consider applications. First we look at the pion PDF at leading twist $\tau=2$ :

$q_{\pi}(x)=(1-x)^{2}\left[\frac{2 f_{\pi}(x)}{1-x}-f_{\pi}^{\prime}(x)\right]=\left[-f_{\pi}(x)(1-x)^{2}\right]^{\prime}$.
Following the pQCD prediction presented in Ref. [21], we consider the parametrization for the pion PDF at the initial scale $\mu_{0}=0.63 \mathrm{GeV}$ as

$$
q_{\pi}\left(x, \mu_{0}\right)=N_{\pi} x^{\alpha-1}(1-x)^{\beta}\left(1+\gamma x^{\delta}\right),
$$

where $N_{\pi}$ is the normalization constant, $\alpha=0.70, \beta=2.03$, $\gamma=13.8, \delta=2$. Notice that in Ref. [27] we derived the LF wave function which produces this PDF. Now we are in a position to fix the profile function $f_{\pi}(x)$, matching Eqs. (34) and (35). Restricting to leading twist, with good accuracy we can use an approximate value of the parameter $\beta \simeq 2$ in Eq. (35). With this and the boundary condition $f_{\pi}(0)=1$ we fix $f_{\pi}(x)$ :

$$
\begin{aligned}
f_{\pi}(x)(1-x)^{2}= & 1-N_{\pi} x^{\alpha}\left[\frac{1}{\alpha}-\frac{2 x}{\alpha+1}+\frac{x^{2}}{\alpha+2}\right. \\
& \left.+\gamma x^{\delta}\left(\frac{1}{\alpha+\delta}-\frac{2 x}{\alpha+\delta+1}+\frac{x^{2}}{\alpha+\delta+2}\right)\right] .
\end{aligned}
$$

It is easy to verify that $f_{\pi}$ obeys the boundary conditions $f_{\pi}(0)=1$ and $f_{\pi}(1)=0$. At large $x$ it scales as $f_{\pi}(x) \sim(1-x)$, which leads to the correct scaling of the pion PDF: $q_{\pi}(x) \sim(1-x)^{2}$. We can also write down the relation of function $f_{\pi}(x)$ with $y_{\pi}(x) \equiv y_{2}(x)$ :

$$
y_{\pi}(x)=1-f_{\pi}(x)(1-x)^{2},
$$

which for large $x$ due to $f_{\pi}(x) \sim(1-x)$ simplifies to

$$
y_{\pi}(x)=1-(1-x)^{3}=x\left(3-3 x+x^{2}\right) .
$$

In Ref. [14] we proposed a formalism for the inclusion of high-Fock states in soft-wall AdS/QCD. In the case of PDF it is given by the sum

$$
q_{\pi}(x)=\sum_{\tau=2,4, \ldots} c_{\tau} q_{\tau}(x),
$$

where $c_{\tau}$ is the set of mixing coefficients defining the partial contributions to the pion PDF, from specific twists $\tau=2,4, \ldots$, which obey the normalization condition

$$
1=\int_{0}^{1} d x q_{\pi}(x)=\sum_{\tau=2,4, \ldots} c_{\tau} \int_{0}^{1} d x q_{\tau}(x)=\sum_{\tau=2,4, \ldots} c_{\tau} .
$$

\section{Nucleon PDFs}

Next we consider the $u$ and $d$ quark PDFs in the nucleon. The nucleon PDFs and GPDs in soft-wall model were calculated for the first time in Ref. [9]. They were extracted from nucleon electromagnetic form factors using an 
integral presentation for the vector field dual to the electromagnetic current in Eq. (25). As we stressed in the Introduction, in previous papers using the soft-wall model, the variable of integration in Eq. (25) was identified with the light-cone variable. It led to the results for the PDF and GPDs with much harder scaling at large $x \rightarrow 1$, i.e., $(1-x)^{\tau-2}$ instead of $(1-x)^{2 \tau-3}$. To solve this problem, one can identify the variable of integration in Eq. (25) with arbitrary function of $x$, i.e., with $y_{\tau}(x)$, and fix $y_{\tau}(x)$ to guarantee the consistency of power scaling of PDFs and GPDs with model-independent results known from QCD. One of the solutions for $y_{\tau}(x)$ consistent with power counting is [25]

$$
y_{\tau}(x)=\exp \left[-\log (1 / x)(1-x)^{1 /(\tau-2)}\right],
$$

leading to the correct large- $x$ scaling of PDFs and GPDs

$$
q_{\tau}(x) \sim \mathcal{H}_{\tau}^{\pi}\left(x, Q^{2}\right) \sim(1-x)^{2 \tau-3} .
$$

As we pointed out before, we follow this novel idea in order to introduce the profile function in the normalization condition for the $z$ profiles of the AdS field dual to the corresponding hadron wave function. Following the pion example considered above, we derive nucleon PDFs starting from the normalization conditions, and consistent with model-independent counting rules. In the nucleon case there are two holographic functions dual to its right$\left(f_{\tau}^{R}\right)$ and left-chirality $\left(f_{\tau}^{L}\right)$ wave functions [Eq. (20)]. The normalization conditions for the $u$ and $d$ quark wave functions, which are equivalent to the normalization conditions for their valence PDFs $\left[u_{v}(x)\right.$ and $\left.d_{v}(x)\right]$ read $u$ quark:

$$
2=\int_{0}^{1} d x u_{v}(x)=\int_{0}^{\infty} d z\left[2 \Phi^{+}(z)+\eta_{u} \partial_{z}\left[z \Phi^{-}(z)\right]\right],
$$

$d$ quark:

$1=\int_{0}^{1} d x d_{v}(x)=\int_{0}^{\infty} d z\left[\Phi^{+}(z)+\eta_{d} \partial_{z}\left[z \Phi^{-}(z)\right]\right]$,

where

$$
\Phi^{ \pm}=\frac{1}{2}\left[\left(f_{\tau}^{R}\right)^{2} \pm\left(f_{\tau}^{L}\right)^{2}\right]
$$

are the combinations of right and left holographic wave functions, $\eta_{u}=2 \eta_{p}+\eta_{n}$ and $\eta_{d}=2 \eta_{n}+\eta_{p}$ are the linear combinations of the nucleon couplings with vector field related to nucleon anomalous magnetic moments $k_{N}$ and fixed as [8,9]: $\eta_{N}=k_{N} \kappa /\left(2 M_{N} \sqrt{2}\right)$, where $M_{N}$ is the nucleon mass.

Notice that the contribution of "nonminimal" terms vanish in the normalization condition for wave functions and PDFs due to gauge invariance, but they contribute to the $x$ dependence of PDFs. Moreover, as seen from Eqs. (43) and (44), the "nonminimal contributions" to the quark PDFs are sufficient to violate the symmetry condition $u_{v}(x) / d_{v}(x)=2$, which occurs at $\eta_{p}=\eta_{n}=0$.

For arbitrary twist the expressions for the quark PDFs in the nucleon are given in Appendix A. For leading twist $\tau=3$, the results for $u_{v}(x)$ and $d_{v}(x)$ read

$$
\begin{aligned}
& u_{v}(x)=\left[-f_{u}(x)(1-x)^{4}\left(1+2 \eta_{u}+(1-x)^{2}\left(1-4 \eta_{u}\right)+2 \eta_{u}(1-x)^{4}\right)\right]^{\prime} \\
& d_{v}(x)=\left[-f_{d}(x)(1-x)^{4}\left(\frac{1}{2}+2 \eta_{d}+(1-x)^{2}\left(\frac{1}{2}-4 \eta_{d}\right)+2 \eta_{d}(1-x)^{4}\right)\right]^{\prime}
\end{aligned}
$$

Both PDFs in Eqs. (46) scale at large $x$ as $(1-x)^{3}$, as dictated by the counting rules $[16,19,20]$, when the $f_{u}(x)$ and $f_{d}(x)$ go to constants independent on $x$. In other words, the Taylor expansion for $f_{q}(x), q=u, d$ has the generic form

$$
f_{q}(x)=\sum_{n} c_{n}(1-x)^{n},
$$

with $\sum_{n} c_{n}=1$, due to the boundary condition $f_{q}(0)=1$. Here the sum over $n$ starts from $n=0$.

World data analysis (see, e.g., Ref. [35]) supports the $(1-x)^{3}$ scaling of the $u_{v}$ PDF, while the extracted $d_{v}$ PDF has softer behavior $(1-x)^{5}$. Note that other groups give either similar fits or softer behavior of the $d$ quark PDF, such as, e.g., $(1-x)^{4.47 \pm 0.55}$ [36], or introduce into the $d$ quark PDF a nontrivial polynomial depending on $\sqrt{x}$ [37]. In our approach we can resolve this puzzle. The solution is based on a suppression of the $(1-x)^{3}$ term in $d_{v}$ [see Eq. (46)], which can occur when the following constraint on the $\eta_{d}$ coupling holds:

$$
\frac{1}{2}+2 \eta_{d}=0
$$

From the latter condition it follows that the dilaton scale parameter $\kappa$ is related to the nucleon mass as $\kappa=0.348 M_{N}=326 \mathrm{MeV}$, which is very close to the value $\kappa=350 \mathrm{MeV}$ used in Refs. [8,9]. Adopting the condition (48) and restricting to the leading order in the $(1-x)$ expansion, we get the following expressions for the quark PDFs in the nucleon: 
$u_{v}(x)=\left[-f_{u}(x)(1-x)^{4}\right]^{\prime}, \quad d_{v}(x)=\left[-f_{d}(x)(1-x)^{6}\right]^{\prime}$.

Now we fix the $u$ and $d$ profile functions $f_{u}(x)$ and $f_{d}(x)$, using predictions for the valence PDFs $u_{v}(x)$ and $d_{v}(x)$ extracted from world data analysis. As an example, we use the results of the MSTW 2008 leading-order (LO) global analysis [35]:

$$
\begin{aligned}
& u_{v}\left(x, \mu_{0}\right)=A_{u} x^{\alpha_{u}-1}(1-x)^{\beta_{u}}\left(1+\epsilon_{u} \sqrt{x}+\gamma_{u} x\right), \\
& d_{v}\left(x, \mu_{0}\right)=A_{d} x^{\alpha_{d}-1}(1-x)^{\beta_{d}}\left(1+\epsilon_{d} \sqrt{x}+\gamma_{d} x\right),
\end{aligned}
$$

where $\mu_{0}=1 \mathrm{GeV}$ is the initial scale. The normalization constants $A_{q}$ and the constants $\alpha_{q}, \beta_{q}, \epsilon_{q}, \gamma_{q}$ were fixed as

$$
\begin{aligned}
A_{u} & =1.4335, \quad A_{d}=5.0903, \\
\alpha_{u} & =0.45232, \quad \alpha_{d}=0.71978, \\
\beta_{u} & =3.0409 \simeq 3, \quad \beta_{d}=5.1244 \simeq 5, \\
\epsilon_{u} & =-2.3737, \quad \epsilon_{d}=-4.3654, \\
\gamma_{u} & =8.9924, \quad \gamma_{d}=7.4730 .
\end{aligned}
$$

Solving the differential equations (49) with the boundary condition $f_{q}(0)=1$ and using $\beta_{u}=2, \beta_{d}=5$ we get

$$
\begin{aligned}
& f_{u}(x)(1-x)^{4} \\
& \quad=1-A_{u} x^{\delta_{u}}\left[B_{u}(x, 0)+\epsilon_{u} \sqrt{x} B_{u}(x, 1 / 2)+\epsilon_{u} x B_{u}(x, 1)\right],
\end{aligned}
$$

$$
\begin{aligned}
& f_{d}(x)(1-x)^{6} \\
& \quad=1-A_{d} x^{\delta_{d}}\left[B_{d}(x, 0)+\epsilon_{d} \sqrt{x} D_{d}(x, 1 / 2)+\epsilon_{d} x B_{d}(x, 1)\right],
\end{aligned}
$$

$$
\begin{aligned}
& \text { where } \\
& \begin{aligned}
B_{u}(x, n) & =\sum_{k=0}^{3} \frac{C_{3}^{k}(-x)^{k}}{\delta_{u}+n+k} \\
& =\frac{1}{\delta_{u}+n}-\frac{3 x}{\delta_{u}+n+1}+\frac{3 x^{2}}{\delta_{u}+n+2}-\frac{x^{3}}{\delta_{u}+n+3},
\end{aligned}
\end{aligned}
$$

$$
\begin{aligned}
B_{d}(x, n)= & \sum_{k=0}^{5} \frac{C_{5}^{k}(-x)^{k}}{\delta_{d}+n+k} \\
= & \frac{1}{\delta_{d}+n}-\frac{5 x}{\delta_{d}+n+1}+\frac{10 x^{2}}{\delta_{d}+n+2} \\
& -\frac{10 x^{3}}{\delta_{d}+n+3}+\frac{5 x^{4}}{\delta_{d}+n+4}-\frac{x^{5}}{\delta_{d}+n+5} .
\end{aligned}
$$

Here $C_{m}^{k}=\frac{m !}{k !(m-k) !}$ are the binomial coefficients. As in the pion case, we derive the relations between sets of nucleon functions $y_{q}(x)$ and $f_{q}(x)$ :

$$
\begin{aligned}
& y_{u}(x)=1-\sqrt{f_{u}(x)}(1-x)^{2}, \\
& y_{d}(x)=1-\sqrt{f_{d}(x)}(1-x)^{3} .
\end{aligned}
$$

For large $x \rightarrow 1$ the expressions for $f_{q}(x)$, and therefore the relations (57), are simplified:

$$
\begin{aligned}
& f_{u}(x)=f_{d}(x)=1, \\
& y_{u}(x)=1-(1-x)^{2}=x(2-x), \\
& y_{d}(x)=1-(1-x)^{3}=x\left(3-3 x+x^{2}\right) .
\end{aligned}
$$

It is clear that in this limit the quark PDFs in the nucleon obey the correct large- $x$ scaling:

$$
u_{v}(x)=8(1-x)^{3}, \quad d_{v}(x)=6(1-x)^{5} .
$$

Note that we use the MSTW 2008 LO global analysis as an example of application of our framework. We can choose any other and match the profile functions $f_{q}$ accordingly. The universality of our approach is that the profile functions $f_{q}$ appear in other parton distributions like TMDs and GPDs. Therefore, as soon as the profile functions $f_{q}$ are fixed from PDFs, one can have predictions for the other parton densities.

Now we turn to a discussion of the magnetization PDFs in nucleons $\mathcal{E}_{v}^{u}(x)$ and $\mathcal{E}_{v}^{d}(x)$. The idea of their derivation is similar to the case of the charged PDFs $u_{v}(x)$ and $d_{v}(x)$. We start with expressions for the contribution to the anomalous magnetic moments $k^{q}$ of $u$ and $d$ quarks in the soft-wall AdS/QCD model [8,9], given as integrals over left- and right-chirality nucleon wave functions with specific twist $\tau$ (20):

$k_{\tau}^{q}=2 M_{N} \eta_{q} \int_{0}^{\infty} d z z \phi_{\tau}^{L}(z) \phi_{\tau}^{R}(z)=\frac{2 M_{N}}{\kappa} \eta_{q} \sqrt{\tau-1}$.

Next we use the integral representation for unity in Eq. (28) and after integration of the holographic variable $z$, we get the magnetization PDFs in the nucleon for leading twist $\tau=3$ (expressions for arbitrary twist can be found in Appendix A):

$$
\mathcal{E}_{v}^{q}(x)=k^{q}\left[-f_{q}(x)(1-x)^{6}\right]^{\prime} .
$$

In principle, the $f_{q}(x)$ profile functions can be different in charged and magnetization PDFs. In the case when they are the same we derive the following relation: 


$$
\frac{\mathcal{E}_{v}^{d}(x)}{d_{v}(x)}=4 \eta_{d} \frac{M_{N}}{\kappa}
$$

\section{TMD}

TMD can arise in soft-wall AdS/QCD by analogy with PDF, using the generalized integral representation for unity, including integration over the longitudinal $x$ and transverse $\mathbf{k}_{\perp}$ variables:

$$
\begin{aligned}
1= & -e^{\kappa^{2} z^{2}} \int_{0}^{1} d\left[f_{\tau}(x) e^{-\kappa^{2} z^{2} /(1-x)^{2}}\right] \\
& \times \int d^{2} \mathbf{k}_{\perp} \frac{D_{\tau}(x)}{\pi \kappa^{2}} e^{-\mathbf{k}_{\perp}^{2} D_{\tau}(x) / \kappa^{2}} \\
= & \frac{e^{\kappa^{2} z^{2}}}{\pi \kappa^{2}} \int_{0}^{1} d x \int d^{2} \mathbf{k}_{\perp}\left[\frac{2 f_{\tau}(x) \kappa^{2} z^{2}}{(1-x)^{3}}-f_{\tau}^{\prime}(x)\right] \\
& \times D_{\tau}(x) e^{-\kappa^{2} z^{2} /(1-x)^{2}} e^{-\mathbf{k}_{\perp}^{2} D_{\tau}(x) / \kappa^{2}}
\end{aligned}
$$

where $D_{\tau}(x)$ is the longitudinal factor derived in Ref. [29], which was fixed from data on the nucleon electromagnetic form factors. The purpose of the function $D_{\tau}(x)$ is to include a running scale in TMD, i.e., a scale parameter, which accompanies the $\mathbf{k}_{\perp}$ dependence in TMDs. In our case the running scale parameter is $\Lambda_{\tau}(x)=\kappa / \sqrt{D_{\tau}(x)}$. As was shown in Ref. [29], the appearance of the $\kappa$ or $M_{N}$ in $\Lambda_{\tau}(x)$ is for convenience, because any different choice can be compensated for by rescaling the function $D_{\tau}(x)$. Such a choice of $\Lambda_{\tau}(x)$ is a generalization of the Gaussian ansatz for TMD with constant scale $\Lambda^{2}=\left\langle\mathbf{k}_{\perp}^{2}\right\rangle$ in the exponential, proposed by the Turin group [38]:

$$
F\left(x, \mathbf{k}_{\perp}\right)=F(x) e^{-\mathbf{k}_{\perp}^{2} /\left\langle\mathbf{k}_{\perp}^{2}\right\rangle} .
$$

This Gaussian ansatz [Eq. (64)] is simple and very useful in practical calculations and analysis of data. However, it is known (see, e.g., Ref. [39]) that it presents difficulties in the description of data on DY processes in some kinematical regions (e.g., at $Q_{\perp} \leq Q$ ). Therefore, the ansatz for the TMD [Eq. (64)] can be crucially checked. In this vein, one can mention results of AdS/QCD and light-front quark models motivated by AdS/QCD (see Refs. [15,26,29]), where it was shown that the hadronic light-front wave functions, PDFs, and TMDs contain scale parameters depending on the light-cone variable $x$, i.e., they can be considered as $x$-dependent scale quantities. It was found in Refs. $[15,26,29]$ that the $x$-dependent scale is crucial for a successful description of data on electromagnetic form factors of nucleons and electroexcitation of nucleon resonances. Also we can see below that our result for the unpolarized quark TMD in the nucleon will contain two terms multiplied with a Gaussian: constant term and term proportional to $\mathbf{k}_{\perp}^{2}$. It is consistent with the form of TMD used by the Pavia group [40]. In the next section we will show that function $D_{\tau}(x)$ can be fixed from expression for the electromagnetic form factor and related to functions $f_{\tau}(x)$ and $y_{\tau}(x)$.

Using the same calculation technique as for the case of PDFs, we insert the integral representation (63) into the normalization condition for the holographic wave function (27) and integrate over the $z$ variable. After that we arrive at the normalization condition for the TMD $F_{\tau}\left(x, \mathbf{k}_{\perp}\right)$, from which the latter can be extracted and expressed through the PDF as

$$
\begin{aligned}
1 & =\int_{0}^{1} d x \int d^{2} \mathbf{k}_{\perp} F_{\tau}\left(x, \mathbf{k}_{\perp}\right), \\
F_{\tau}\left(x, \mathbf{k}_{\perp}\right) & =q_{\tau}(x) \frac{D_{\tau}(x)}{\pi \kappa^{2}} e^{-\mathbf{k}_{\perp}^{2} D_{\tau}(x) / \kappa^{2}} .
\end{aligned}
$$

Also it is important to stress that from the results for generic PDFs and TMDs derived in the present paper one can set up a LF quark model in analogy with our previous papers $[26,29]$. In particular, the LF wave function for generic hadron with twist $\tau$ reads

$$
\psi\left(x, \mathbf{k}_{\perp}\right)=\frac{4 \pi}{\kappa} \sqrt{q_{\tau}(x) D_{\tau}(x)} \exp \left[-\frac{\mathbf{k}_{\perp}^{2}}{2 \kappa^{2}} D_{\tau}(x)\right] .
$$

Note that generic TMD and PDF are expressed in term of the LF wave function (66) as

$$
\begin{aligned}
F_{\tau}\left(x, \mathbf{k}_{\perp}\right) & =\frac{1}{16 \pi^{3}}\left|\psi\left(x, \mathbf{k}_{\perp}\right)\right|^{2}, \\
q_{\tau}(x) & =\int \frac{d^{2} \mathbf{k}_{\perp}}{16 \pi^{3}}\left|\psi\left(x, \mathbf{k}_{\perp}\right)\right|^{2}=\int d^{2} \mathbf{k}_{\perp} F_{\tau}\left(x, \mathbf{k}_{\perp}\right) .
\end{aligned}
$$

Now let us consider as example the result for the unpolarized quark TMD in nucleon $f_{1}^{q_{v}}\left(x, \mathbf{k}_{\perp}\right)$. As in the case of the PDF it is contributed by two wave functions $\phi^{R}(z)$ and $\phi^{L}(z)$ [Eq. (20)] corresponding to the leading and subleading twist or having orbital moment $L=0$ and $L=1$. The $\phi^{R}(z)$ function generates the contribution to TMD $f_{1, R}^{q_{v}}\left(x, \mathbf{k}_{\perp}\right)$ fixed from condition similar to Eq. (65), while the $\phi^{L}(z)$ gives the contribution $f_{1, L}^{q_{v}}\left(x, \mathbf{k}_{\perp}\right)$ proportional to $\mathbf{k}_{\perp}^{2}$ :

$$
f_{1}^{q_{v}}\left(x, \mathbf{k}_{\perp}\right)=f_{1, R}^{q_{v}}\left(x, \mathbf{k}_{\perp}\right)+f_{1, L}^{q_{v}}\left(x, \mathbf{k}_{\perp}\right),
$$

where

$$
\begin{aligned}
& f_{1, R}^{q_{v}}\left(x, \mathbf{k}_{\perp}\right)=q_{v}^{+}(x) \frac{D_{q}(x)}{2 \pi \kappa^{2}} e^{-\mathbf{k}_{\perp}^{2} D_{q}(x) / \kappa^{2}}, \\
& f_{1, L}^{q_{v}}\left(x, \mathbf{k}_{\perp}\right)=q_{v}^{-}(x) \frac{\mathbf{k}_{\perp}^{2} D_{q}^{2}(x)}{2 \pi \kappa^{4}} e^{-\mathbf{k}_{\perp}^{2} D_{q}(x) / \kappa^{2}} .
\end{aligned}
$$


Here $q_{v}^{ \pm}(x)=q_{v}(x) \pm \delta q_{v}(x), q_{v}(x)$ and $\delta q_{v}(x)$ are the helicity-independent and helicity-dependent valence quark parton distributions. As we mentioned before, the form of our expression for TMD

$f_{1}^{q_{v}}\left(x, \mathbf{k}_{\perp}\right)=\left[q_{v}^{+}(x)+q_{v}^{-}(x) \frac{\mathbf{k}_{\perp}^{2} D_{q}(x)}{\kappa^{2}}\right] \frac{D_{q}(x)}{2 \pi \kappa^{2}} e^{-\mathbf{k}_{\perp}^{2} D_{q}(x) / \kappa^{2}}$

is very similar to the parametrization used by the Pavia group [40]:

$$
f_{1}^{a}\left(x, \mathbf{k}_{\perp}\right)=\frac{1}{\pi g_{1 a}} \frac{1+\lambda \mathbf{k}_{\perp}^{2}}{1+\lambda g_{1 a}} e^{-\mathbf{k}_{\perp}^{2} / g_{1 a}} .
$$

Using expressions for nucleon PDFs and TMDs one can set up the LF wave functions for the nucleon following Refs. [26,29]:

$$
\begin{aligned}
\psi_{ \pm q}^{ \pm}\left(x, \mathbf{k}_{\perp}\right) & =\varphi_{q}^{(1)}\left(x, \mathbf{k}_{\perp}\right), \\
\psi_{\mp q}^{ \pm}\left(x, \mathbf{k}_{\perp}\right) & =\mp \frac{k^{1} \pm i k^{2}}{M_{N}} \varphi_{q}^{(2)}\left(x, \mathbf{k}_{\perp}\right),
\end{aligned}
$$

where

$$
\begin{gathered}
\varphi_{q}^{(1)}\left(x, \mathbf{k}_{\perp}\right)=\frac{2 \pi \sqrt{2}}{\kappa} \sqrt{q_{v}^{+}(x) D_{q}(x)} \exp \left[-\frac{\mathbf{k}_{\perp}^{2}}{2 \kappa^{2}} D_{q}(x)\right], \\
\frac{1}{M_{N}} \varphi_{q}^{(2)}\left(x, \mathbf{k}_{\perp}\right)=\frac{2 \pi c_{q} \sqrt{2}}{\kappa^{2}} \sqrt{q_{v}^{-}(x)} D_{q}(x) \exp \left[-\frac{\mathbf{k}_{\perp}^{2}}{2 \kappa^{2}} D_{q}(x)\right] .
\end{gathered}
$$

Here $c_{u}=1, c_{d}=-1, \psi_{\lambda_{q} q}^{\lambda_{N}}\left(x, \mathbf{k}_{\perp}\right)$ are the light-front wave functions at the initial scale $\mu_{0}$ with specific helicities for the nucleon $\lambda_{N}= \pm$ and for the struck quark $\lambda_{q}= \pm$, where the plus and minus correspond to $+\frac{1}{2}$ and $-\frac{1}{2}$, respectively. Note, in terms of the LF wave functions (72) the unpolarized quark TMD in nucleon reads [41]

$$
\begin{aligned}
f_{1}^{q_{v}}\left(x, \mathbf{k}_{\perp}\right) & =\frac{1}{16 \pi^{3}}\left[\left|\psi_{+q}^{+}\left(x, \mathbf{k}_{\perp}\right)\right|^{2}+\left|\psi_{-q}^{+}\left(x, \mathbf{k}_{\perp}\right)\right|^{2}\right] \\
& =\frac{1}{16 \pi^{3}}\left[\left(\varphi_{q}^{(1)}\left(x, \mathbf{k}_{\perp}\right)\right)^{2}+\frac{\mathbf{k}_{\perp}^{2}}{M_{N}^{2}}\left(\varphi_{q}^{(2)}\left(x, \mathbf{k}_{\perp}\right)\right)^{2}\right] .
\end{aligned}
$$

Note $q_{v}^{ \pm}(x)$ and $\mathcal{E}_{v}^{q}(x)$ PDFs are related as [29]

$$
\mathcal{E}_{v}^{q}(x)=c_{q} \sqrt{q_{v}^{+}(x) q_{v}^{-}(x) D_{q}(x)}(1-x) .
$$

The full set of the valence $T$-even TMDs generated by LF wave functions derived above is listed in Appendix B.

\section{GPD}

As we mentioned before, the nucleon GPDs were calculated for the first time in soft-wall AdS/QCD in Ref. [9]. These quantities were expressed in terms of generalized light-cone variable $y_{\tau}(x)$, which has direct relation to the profile function $f_{\tau}(x)$. Function $f_{\tau}(x)$ is more convenient for displaying power behavior of hadronic parton distributions (PDFs, TMDs, and GPDs). In particular, for arbitrary twist $\tau$, a generic GPD in hadron reads [26]

$$
\begin{aligned}
\mathcal{H}_{\tau}\left(y_{\tau}(x), Q^{2}\right) & =(\tau-1)\left(1-y_{\tau}(x)\right)^{\tau-2}\left[y_{\tau}(x)\right]^{a}, \\
a & =\frac{Q^{2}}{4 \kappa^{2}} .
\end{aligned}
$$

It can be written in more convenient form in terms of the PDF:

$\mathcal{H}_{\tau}\left(x, Q^{2}\right)=q_{\tau}(x)\left[y_{\tau}(x)\right]^{a}=q_{\tau}(x) \exp \left(-a \log \left[1 / y_{\tau}(x)\right]\right)$,

where the PDF $q_{\tau}(x)$ and light-cone function $y_{\tau}(x)$ are expressed through profile function $f_{\tau}(x)$ according to Eqs. (33) and (30).

Next we constrain function $D_{\tau}(x)$ and relate it to functions $y_{\tau}(x)$ and $f_{\tau}(x)$ matching the expression for the hadronic form factors in two approaches-soft-wall AdS/QCD and LF QCD. The LF QCD result for the hadron form factor is given by the DYW formula [16]

$$
F_{\tau}\left(Q^{2}\right)=\int_{0}^{1} d x \int \frac{d^{2} \mathbf{k}_{\perp}}{16 \pi^{3}} \psi_{\tau}^{\dagger}\left(x, \mathbf{k}_{\perp}^{\prime}\right) \psi_{\tau}\left(x, \mathbf{k}_{\perp}\right),
$$

where $\psi\left(x, \mathbf{k}_{\perp}\right) \equiv \psi\left(x, \mathbf{k}_{\perp} ; \mu_{0}\right)$ is wave function derived in Eq. (66), $\mathbf{k}_{\perp}^{\prime}=\mathbf{k}_{\perp}+(1-x) \mathbf{q}_{\perp}$, and $Q^{2}=\mathbf{q}_{\perp}^{2}$.

We get

$$
\begin{aligned}
F_{\tau}\left(Q^{2}\right) & =\int_{0}^{1} d x q_{\tau}(x) \exp \left[-a \log \left[1 / y_{\tau}(x)\right]\right] \\
& =\int_{0}^{1} d x q_{\tau}(x) \exp \left[-a D_{\tau}(x)(1-x)^{2}\right]
\end{aligned}
$$

or

$$
\begin{aligned}
D_{\tau}(x) & =\frac{1}{(1-x)^{2}} \log \left[1 / y_{\tau}(x)\right] \\
& =\frac{1}{(1-x)^{2}} \log \left[1-\left(f_{\tau}(x)\right)^{\frac{1}{\tau-1}}(1-x)^{2}\right]^{-1} .
\end{aligned}
$$

For a large- $x$ function $D_{\tau}(x)$ behaves as

$$
D_{\tau}(x)=\left(f_{\tau}(x)\right)^{\frac{1}{\tau-1}},
$$

where $f_{\pi}(x)=1-x, f_{u}(x)=f_{d}(x)=1$ and therefore $D_{\pi}(x)=1-x, D_{u}(x)=D_{d}(x)=1$. It leads to the following scaling of the TMDs at large $x$ : 


$$
f_{1}^{\pi}\left(x, \mathbf{k}_{\perp}\right)=q_{\pi}(x)(1-x) \frac{e^{-\mathbf{k}_{\perp}^{2}(1-x) / \kappa^{2}}}{\pi \kappa^{2}}
$$

for pion,

$$
f_{1}^{q_{v}}\left(x, \mathbf{k}_{\perp}\right)=\left[q_{v}^{+}(x)+q_{v}^{-}(x) \frac{\mathbf{k}_{\perp}^{2}}{\kappa^{2}}\right] \frac{e^{-\mathbf{k}_{\perp}^{2} / \kappa^{2}}}{2 \pi \kappa^{2}}
$$

for nucleon.

Now we consider specific cases for GPDs. In the pion case we have $\tau=2$ and $y_{\pi}(x)=1-f_{\pi}(x)(1-x)^{2}$, where the pion profile function $f_{\pi}(x)$ is fixed from pion PDF by Eq. (36). The pion PDF $q_{\pi}(x)$ is fixed from the data. Therefore, we give the pion GPD prediction at the initial scale $\mu_{0}=1 \mathrm{GeV}$ in terms of the pion PDF, or more precisely in terms of constants parametrizing $\operatorname{PDF}\left(N_{\pi}, \alpha\right.$, $\beta, \gamma, \delta$ ) fixed in Ref. [21]. At large $x$ the profile functions $f_{\pi}(x) \rightarrow(1-x)$ and $y_{\pi}(x) \rightarrow 1$ [see Eq. (38)], and the scaling of our result for the pion GPD $(1-x)^{2}$ is consistent with the pQCD prediction [20]: it coincides with the leading-order result for the pion PDF and is independent of $Q^{2}$ :

$$
\mathcal{H}_{\pi}\left(x, Q^{2}\right)=q_{\pi}(x)=3(1-x)^{2} .
$$

In the nucleon case we have $\tau=3, y_{u}(x)=$ $1-\sqrt{f_{u}(x)}(1-x)^{2}$, and $y_{d}(x)=1-\sqrt{f_{d}(x)}(1-x)^{3}$. The quark profile functions $f_{u}(x)$ and $f_{d}(x)$ are fixed from the corresponding nucleon PDFs extracted from global data analysis at the initial scale $\mu_{0}=1 \mathrm{GeV}$ [35]. The four (charged and magnetization) nucleon GPDs at the initial scale $\mu_{0}=1 \mathrm{GeV}$ are defined as

$$
\begin{aligned}
\mathcal{H}_{v}^{q}\left(x, Q^{2}\right) & =q_{v}(x)\left[y_{q}(x)\right]^{a}, \\
\mathcal{E}_{v}^{q}\left(x, Q^{2}\right) & =\mathcal{E}_{v}^{q}(x)\left[y_{q}(x)\right]^{a} .
\end{aligned}
$$

Finally we consider the limit of large $x$. In this case the profile functions $f_{q}(x)$ and functions $y_{q}(x)$ approach 1: $f_{u}(x)=f_{d}(x)=1$ and $y_{u}(x)=y_{d}(x)=1$ [see Eq. (58)]. The scaling of the nucleon charge and magnetization GPDs are also (as in case of pion) consistent with the pQCD predictions [20]:

$$
\begin{aligned}
\mathcal{H}_{v}^{u}\left(x, Q^{2}\right) & =u_{v}(x)=8(1-x)^{3}, \\
\mathcal{E}_{v}^{q}\left(x, Q^{2}\right) & =\mathcal{E}_{v}^{q}(x)=6 \mathcal{E}_{v}^{q}(1-x)^{5} .
\end{aligned}
$$

In the case of the $d$ quark charge GPD $\mathcal{H}_{v}^{d}\left(x, Q^{2}\right)$ we have two possibilities at large $x$. In general it scales as $(1-x)^{3}$ in agreement with pQCD [20]. On the other hand, if we suppress the leading-order term $(1-x)^{3}$ in the $d$ quark PDF using the constraint (48), then $d_{v}(x)$ has softer $(1-x)^{5}$ behavior consistent with result of world data analysis [35]. In this vein, we also get $(1-x)^{5}$ scaling of the $\mathcal{H}_{v}^{d}\left(x, Q^{2}\right)$. Note that the large- $x$ scaling of the pion and nucleon GPDs is governed by corresponding PDFs.

\section{SUMMARY}

In the present paper we explicitly demonstrated how to correctly define the hadronic parton distributions (PDFs, TMDs, and GPDs) in the soft-wall AdS/QCD approach based on the use of quadratic dilaton. The large- $x$ behavior of PDFs and GPDs is consistent with model-independent counting rules. For the first time, we derived results for the large- $x$ behavior of TMDs. Our predictions for the $T$-even TMDs of nucleon are listed in Appendix B. All parton distributions are defined in terms of profile functions $f_{\tau}(x)$ depending on the light-cone coordinate. The functions $f_{\tau}(x)$ are related to the PDFs and obey the boundary condition $f_{\tau}(0)=1$. We also proposed a solution to the puzzle related with a softer large- $x$ behavior of the valence $d$ quark PDF in nucleon in comparison with the one of the $u$ quark. It can be obtained due to the vanishing of the leading-order term $(1-x)^{3}$ when nonminimal couplings of the nucleons with the electromagnetic field obey the condition (48). Profile functions are fixed from data analysis on PDFs and can then be tested in the phenomenology of TMDs and GPDs.

\section{ACKNOWLEDGMENTS}

This work was funded by "Verbundprojekt 05P2018Ausbau von ALICE am LHC: Jets und partonische Struktur von Kernen" (Förderkennzeichen: 05P18VTCA1), by "Verbundprojekt 05A2017-CRESST-XENON: Direkte Suche nach Dunkler Materie mit XENON1T/nT und CRESST-III. Teilprojekt 1" (Förderkennzeichen 05A17VTA)", by CONICYT (Chile) under Grants No. 7912010025, No. 1180232, and No. ANID PIA/ APOYO AFB180002, and by FONDECYT (Chile) under Grant No. 1191103.

\section{APPENDIX A: USEFUL ANALYTICAL RESULTS FOR PARTON DENSITIES}

For arbitrary twist the expressions for the quark PDFs in nucleon read

$$
\begin{gathered}
u_{v}(x)=\left[-f_{u}(x)(1-x)^{2(\tau-1)}\left(1+\eta_{u}(\tau-1)+(1-x)^{2}\left(1-2 \eta_{u}(\tau-1)\right)+\eta_{u}(\tau-1)(1-x)^{4}\right)\right]^{\prime}, \\
d_{v}(x)=\left[-f_{d}(x)(1-x)^{2(\tau-1)}\left(\frac{1}{2}+\eta_{d}(\tau-1)+(1-x)^{2}\left(\frac{1}{2}-2 \eta_{d}(\tau-1)\right)+\eta_{d}(\tau-1)(1-x)^{4}\right)\right]^{\prime} .
\end{gathered}
$$


In the $\tau=3$ case and using the additional constraint $2 \eta_{d}=-1 / 2$ (it means that we get $2 \eta_{u}=3 \eta_{p}-1 / 4$ ), we can suppress the leading $(1-x)^{3}$ term in $d_{v}(x)$. Therefore, $d_{v}(x)$ dominates by the next-to-leading term $(1-x)^{5}$. Taking all these arguments into account we arrive at

$$
\begin{gathered}
u_{v}(x)=\left[-\frac{3}{4} f_{u}(x)(1-x)^{4}\left(1+4 \eta_{p}+2(1-x)^{2}\left(1-4 \eta_{p}\right)-\frac{1}{3}(1-x)^{4}\left(1-12 \eta_{p}\right)\right)\right]^{\prime}, \\
d_{v}(x)=\left[-\frac{3}{2} f_{d}(x)(1-x)^{6}\left(1-\frac{(1-x)^{2}}{3}\right)\right]^{\prime} .
\end{gathered}
$$

Restricting for simplicity to the leading order in $(1-x)$ expansion of $u_{v}(x)$ and $d_{v}(x)$ we finally get

$$
u_{v}(x)=\left[-f_{u}(x)(1-x)^{4}\right]^{\prime}, \quad d_{v}(x)=\left[-f_{d}(x)(1-x)^{6}\right]^{\prime} .
$$

Magnetization quark PDFs in nucleon for arbitrary twist are given by

$$
\mathcal{E}_{v}^{q}(x)=k^{q}\left[-f_{q}(x)(1-x)^{2 \tau}\right]^{\prime}, \quad k^{q}=\frac{2 M_{N}}{\kappa} \eta_{q} \sqrt{\tau-1} .
$$

\section{APPENDIX B: $T$-EVEN TMDS OF NUCLEON}

Here we list the $T$-even TMDs of nucleon using the derived LF decomposition discussed in [29,41] and wave functions derived in Eq. (72):

$$
\begin{aligned}
f_{1}^{q_{v}}\left(x, \mathbf{k}_{\perp}\right) & \equiv h_{1 T}^{q_{v}}\left(x, \mathbf{k}_{\perp}\right)=\frac{1}{16 \pi^{3}}\left[\left(\varphi_{q}^{(1)}\left(x, \mathbf{k}_{\perp}\right)\right)^{2}+\frac{\mathbf{k}_{\perp}^{2}}{M_{N}^{2}}\left(\varphi_{q}^{(2)}\left(x, \mathbf{k}_{\perp}\right)\right)^{2}\right], \\
g_{1 L}^{q_{v}}\left(x, \mathbf{k}_{\perp}\right) & =\frac{1}{16 \pi^{3}}\left[\left(\varphi_{q}^{(1)}\left(x, \mathbf{k}_{\perp}\right)\right)^{2}-\frac{\mathbf{k}_{\perp}^{2}}{M_{N}^{2}}\left(\varphi_{q}^{(2)}\left(x, \mathbf{k}_{\perp}\right)\right)^{2}\right], \\
g_{1 T}^{q_{v}}\left(x, \mathbf{k}_{\perp}\right) & \equiv-h_{1 L}^{\perp q_{v}}\left(x, \mathbf{k}_{\perp}\right)=\frac{1}{8 \pi^{3}} \varphi_{q}^{(1)}\left(x, \mathbf{k}_{\perp}\right) \varphi_{q}^{(2)}\left(x, \mathbf{k}_{\perp}\right), \\
h_{1}^{q_{v}}\left(x, \mathbf{k}_{\perp}\right) & \equiv h_{1 T}^{q_{v}}\left(x, \mathbf{k}_{\perp}\right)+\frac{\mathbf{k}_{\perp}^{2}}{2 M_{N}^{2}} h_{1 T}^{\perp q_{v}}\left(x, \mathbf{k}_{\perp}\right)=\frac{1}{16 \pi^{3}}\left(\varphi_{q}^{(1)}\left(x, \mathbf{k}_{\perp}\right)\right)^{2}, \\
\frac{\mathbf{k}_{\perp}^{2}}{2 M_{N}^{2}} h_{1 T}^{\perp q_{v}}\left(x, \mathbf{k}_{\perp}\right) & =\frac{1}{2}\left[g_{1 L}^{q_{v}}\left(x, \mathbf{k}_{\perp}\right)-f_{1}^{q_{v}}\left(x, \mathbf{k}_{\perp}\right)\right]=g_{1 L}^{q_{v}}\left(x, \mathbf{k}_{\perp}\right)-h_{1}^{q_{v}}\left(x, \mathbf{k}_{\perp}\right)=-\frac{\mathbf{k}_{\perp}^{2}}{16 \pi^{3} M_{N}^{2}}\left(\varphi_{q}^{(2)}\left(x, \mathbf{k}_{\perp}\right)\right)^{2} .
\end{aligned}
$$

Using our expressions of the LF wave functions we express TMDs through the PDFs

$$
\begin{aligned}
f_{1}^{q_{v}}\left(x, \mathbf{k}_{\perp}\right) & \equiv h_{1 T}^{q_{v}}\left(x, \mathbf{k}_{\perp}\right)=\mathcal{F}_{1}\left(x, \mathbf{k}_{\perp}\right)+\mathcal{F}_{2}\left(x, \mathbf{k}_{\perp}\right), \\
g_{1 L}^{q_{v}}\left(x, \mathbf{k}_{\perp}\right) & =\mathcal{F}_{1}\left(x, \mathbf{k}_{\perp}\right)-\mathcal{F}_{2}\left(x, \mathbf{k}_{\perp}\right), \\
g_{1 T}^{q_{v}}\left(x, \mathbf{k}_{\perp}\right) & \equiv-h_{1 L}^{\perp q_{v}}\left(x, \mathbf{k}_{\perp}\right)=\mathcal{F}_{3}\left(x, \mathbf{k}_{\perp}\right), \\
h_{1}^{q_{v}}\left(x, \mathbf{k}_{\perp}\right) & =\mathcal{F}_{1}\left(x, \mathbf{k}_{\perp}\right), \\
\frac{\mathbf{k}_{\perp}^{2}}{2 M_{N}^{2}} h_{1 T}^{\perp q_{v}}\left(x, \mathbf{k}_{\perp}\right) & =-\mathcal{F}_{2}\left(x, \mathbf{k}_{\perp}\right),
\end{aligned}
$$

where

$$
\begin{aligned}
& \mathcal{F}_{1}\left(x, \mathbf{k}_{\perp}\right)=q_{v}^{+}(x) \frac{D_{q}(x)}{2 \pi \kappa^{2}} e^{-\frac{\mathbf{k}_{\perp}^{2}}{\kappa^{2}} D_{q}(x)}, \\
& \mathcal{F}_{2}\left(x, \mathbf{k}_{\perp}\right)=q_{v}^{-}(x) \frac{\mathbf{k}_{\perp}^{2} D_{q}^{2}(x)}{2 \pi \kappa^{4}} e^{-\frac{\mathbf{k}_{\perp}^{2}}{\kappa^{2}} D_{q}(x)}, \\
& \mathcal{F}_{3}\left(x, \mathbf{k}_{\perp}\right)=c_{q} \sqrt{\frac{4 \kappa^{2}}{\mathbf{k}_{\perp}^{2}} \mathcal{F}_{1}\left(x, \mathbf{k}_{\perp}\right) \mathcal{F}_{2}\left(x, \mathbf{k}_{\perp}\right)}=\sqrt{q_{v}^{+}(x) q_{v}^{-}(x)} \frac{c_{q} D_{q}^{3 / 2}(x)}{\pi \kappa^{2}} e^{-\frac{\mathbf{k}_{\perp}^{2}}{\kappa^{2}} D_{q}(x)} .
\end{aligned}
$$


Performing the $\mathbf{k}_{\perp}$ integration over the TMDs with

$$
\operatorname{TMD}(x)=\int d^{2} \mathbf{k}_{\perp} \operatorname{TMD}\left(x, \mathbf{k}_{\perp}\right), \quad \overline{\operatorname{TMD}}(x)=\int d^{2} \mathbf{k}_{\perp} \frac{\mathbf{k}_{\perp}^{2}}{2 M_{N}^{2}} \operatorname{TMD}\left(x, \mathbf{k}_{\perp}\right)
$$

gives the identities

$$
\begin{array}{ll}
f_{1}^{q_{v}}(x) \equiv h_{1 T}^{q_{v}}(x)=q_{v}(x), & g_{1 L}^{q_{v}}(x)=\delta q_{v}(x), \quad g_{1 T}^{q_{v}}(x) \equiv-h_{1 L}^{\perp q_{v}}(x)=\frac{\mathcal{E}^{q}(x)}{1-x}, \\
h_{1}^{q_{v}}(x)=\frac{q_{v}(x)+\delta q_{v}(x)}{2}, & h_{1 T}^{\perp q_{v}}(x)=-\frac{q_{v}(x)-\delta q_{v}(x)}{2}
\end{array}
$$

The integration over $x$ leads to the normalization conditions

$$
\int_{0}^{1} d x f_{1}^{q_{v}}(x)=\int_{0}^{1} d x h_{1 T}^{q_{v}}(x)=n_{q}, \quad \int_{0}^{1} d x g_{1 L}^{q_{v}}(x)=g_{A}^{q}, \quad \int_{0}^{1} d x h_{1}^{q_{v}}(x)=g_{T}^{q},
$$

where $n_{q}$ is the number of $u$ or $d$ valence quarks in the proton, $g_{A}^{q}$ is the axial charge of a quark with flavor $q=u$ or $d$, and $g_{T}^{q}$ is the tensor charge. Our TMDs satisfy all relations and inequalities found before in theoretical approaches (see the detailed discussion in Ref. [29]).

[1] A. Karch, E. Katz, D. T. Son, and M. A. Stephanov, Phys. Rev. D 74, 015005 (2006).

[2] S. J. Brodsky and G. F. de Teramond, Phys. Rev. Lett. 96, 201601 (2006).

[3] O. Andreev, Phys. Rev. D 73, 107901 (2006).

[4] S. J. Brodsky, G. F. de Teramond, H. G. Dosch, and J. Erlich, Phys. Rep. 584, 1 (2015).

[5] T. Gherghetta, J. I. Kapusta, and T. M. Kelley, Phys. Rev. D 79, 076003 (2009).

[6] M. Jarvinen and E. Kiritsis, J. High Energy Phys. 03 (2012) 002.

[7] S. J. Brodsky and G. F. de Teramond, Phys. Rev. D 77, 056007 (2008).

[8] Z. Abidin and C.E. Carlson, Phys. Rev. D 79, 115003 (2009).

[9] A. Vega, I. Schmidt, T. Gutsche, and V. E. Lyubovitskij, Phys. Rev. D 83, 036001 (2011).

[10] T. Branz, T. Gutsche, V. E. Lyubovitskij, I. Schmidt, and A. Vega, Phys. Rev. D 82, 074022 (2010).

[11] S. J. Brodsky, F. G. Cao, and G. F. de Teramond, Phys. Rev. D 84, 075012 (2011).

[12] T. Gutsche, V. E. Lyubovitskij, I. Schmidt, and A. Vega, Phys. Rev. D 85, 076003 (2012).

[13] T. Gutsche, V. E. Lyubovitskij, and I. Schmidt, Nucl. Phys. B952, 114934 (2020); T. Gutsche, V. E. Lyubovitskij, I. Schmidt, and A. Vega, Phys. Rev. D 87, 016017 (2013).

[14] T. Gutsche, V. E. Lyubovitskij, I. Schmidt, and A. Vega, Phys. Rev. D 86, 036007 (2012); 91, 114001 (2015).

[15] T. Gutsche, V. E. Lyubovitskij, and I. Schmidt, Phys. Rev. D 94, 116006 (2016); 97, 054011 (2018); 101, 034026 (2020).

[16] S. D. Drell and T. M. Yan, Phys. Rev. Lett. 24, 181 (1970).
[17] E. D. Bloom and F. J. Gilman, Phys. Rev. Lett. 25, 1140 (1970).

[18] S. J. Brodsky and G. R. Farrar, Phys. Rev. Lett. 31, 1153 (1973); V. A. Matveev, R. M. Muradyan, and A. N. Tavkhelidze, Teor. Mat. Fiz. 15, 332 (1973) [Lett. Nuovo Cimento 5, 907 (1972)].

[19] R. Blankenbecler and S. J. Brodsky, Phys. Rev. D 10, 2973 (1974).

[20] F. Yuan, Phys. Rev. D 69, 051501 (2004).

[21] M. Aicher, A. Schafer, and W. Vogelsang, Phys. Rev. Lett. 105, 252003 (2010).

[22] J. S. Conway, C. E. Adolphsen, J. P. Alexander, K. J. Anderson, J. G. Heinrich, J. E. Pilcher, A. Possoz, E. I. Rosenberg et al., Phys. Rev. D 39, 92 (1989).

[23] H. W. Lin et al., arXiv:2006.08636.

[24] A. Vega, I. Schmidt, T. Branz, T. Gutsche, and V.E. Lyubovitskij, Phys. Rev. D 80, 055014 (2009).

[25] V.E. Lyubovitskij, in Proceedings at the International Conference Venturing off the Lightcone-Local versus Global Features (Light Cone 2013), Skiathos, Greece, 2013 (2013), http://www.tp2.ruhr-uni-bochum.de/ lc2013/ downloads/talks/Lyubovitsky-LC2013.pdf; V. E. Lyubovitskij, T. Gutsche, I. Schmidt, and A. Vega, Few-Body Syst. 55, 447 (2014).

[26] T. Gutsche, V. E. Lyubovitskij, I. Schmidt, and A. Vega, Phys. Rev. D 89, 054033 (2014); 92, 019902(E) (2015); A. Vega, I. Schmidt, T. Gutsche, and V.E. Lyubovitskij, arXiv:1306 .1597.

[27] T. Gutsche, V. E. Lyubovitskij, I. Schmidt, and A. Vega, J. Phys. G 42, 095005 (2015).

[28] T. Gutsche, V. E. Lyubovitskij, I. Schmidt, and A. Vega, Phys. Rev. D 91, 054028 (2015). 
[29] T. Gutsche, V. E. Lyubovitskij, and I. Schmidt, Eur. Phys. J. C 77, 86 (2017).

[30] G. F. de Teramond, T. Liu, R. S. Sufian, H. G. Dosch, S. J. Brodsky, and A. Deur (HLFHS Collaboration), Phys. Rev. Lett. 120, 182001 (2018).

[31] S. J. Brodsky, G. F. de Teramond, and H. G. Dosch, arXiv: 2004.07756.

[32] A. Vega and M. A. Martin Contreras, arXiv:2005.04501.

[33] L. Chang, K. Raya, and X. Wang, arXiv:2001.07352.

[34] H. R. Grigoryan and A. V. Radyushkin, Phys. Rev. D 76, 095007 (2007).

[35] A. D. Martin, W. J. Stirling, R. S. Thornem, and G. Watt, Eur. Phys. J. C 63, 189 (2009).
[36] S. Alekhin, J. Blümlein, S. Moch, and R. Placakyte, Phys. Rev. D 96, 014011 (2017).

[37] T. J. Hou et al., arXiv:1912.10053.

[38] M. Anselmino, U. D’Alesio, and F. Murgia, Phys. Rev. D 67, 074010 (2003).

[39] A. Bacchetta, G. Bozzi, M. Lambertsen, F. Piacenza, J. Steiglechner, and W. Vogelsang, Phys. Rev. D 100, 014018 (2019).

[40] A. Bacchetta, F. Delcarro, C. Pisano, M. Radici, and A. Signori, J. High Energy Phys. 06 (2017) 081; 06 (2019) 051(E).

[41] A. Bacchetta, F. Conti, and M. Radici, Phys. Rev. D 78, 074010 (2008). 